\title{
A Simulation Model of the Decomposition Activity of Multiple Fungi
}

\author{
Ruoxuan Liu ${ }^{1, *}$ Jianhui $\mathrm{Ye}^{2, *}$ \\ ${ }^{1}$ Zhejiang University, Agriculture and Biotechnology College,Xihu District, Hangzhou, Zhejiang Province, China \\ ${ }^{2}$ Zhejiang University, Agriculture and Biotechnology College,Xihu District, Hangzhou, Zhejiang Province,China
}

\begin{abstract}
Fungi play an essential role in carbon cycle by decomposing the litter and woody fibers. This study aims to establish a model for describing the decomposition by multiple fungal activities in different environment. We establish Colony Distribution-Decomposition Model to simulate the interaction between various fungi and describe the relationship between fungal activity and decomposition, which is divided into three sub-models: single colony growth and extension model, decomposition model and competition model. We applied this model to the study of the interaction of different species, the sensitivity of fungi to environmental changes in short-term and long-term, the adapatability to different climate types for various species and the role biodiversity plays in the breakdown of the litter. The developed model has the advantages of comprehensiveness, stability, rationality, and wide application, which is a feasible and reasonable model to assist the analysis on the interaction between multiple fungi and describe the relationship between fungal activity and decomposition.
\end{abstract}

\section{Introduction}

The carbon cycle refers to the phenomenon in which carbon elements on the earth exchange and cycle. It is of great significance to maintain the stability of the earth' $\mathrm{s}$ ecological environment. As the main decomposer in terrestrial ecosystems, fungi are crucial to the carbon cycle. In the biosphere, carbon mainly exists in the form of organic compounds, such as plant material and woody fibers. Fungi participate in the carbon cycle through their decomposition activity.

Many studies indicate that the growth and decomposition rate of fungi are closely related with environment factors including temperature and humidity. It is meaningful to explore the relationship between lignin decomposition rate and fungal characteristics. The sensitivity of lignin decomposition rate to environmental changes is important for application of fungi to promote the carbon cycle and maintain ecosystem stability.

In the present study, we built a mathematical model for describing the decomposition rate of ground litter and woody fibers through the interaction of different fungi.

\section{Preparations}

\subsection{Data Research}

we search for the online data, including 34 fungi in 20 species ${ }^{[1][2]}$, which describes the characteristics of different fungal species, including the rate of mycelial extension, the amount of lignin decomposition in a certain period of time, the optimum temperature and humidity for fungal growth.

\subsection{Assumptions and Justifications}

1.Ignore the difference in decomposition rate caused by the difference in litter

2.Assume the lignin is limited and the lignin distribution is uniform.

3.The colony shape can be approximated as a standard circle.

4.Competitive effect or the amount of lignin left only reduce the number of the fungi at a certain point but will not affect the expansion.

5.To simplify the model, we ignore the difference between aerial hyphae and vegetative hyphae, which means all the cells on the hyphae can decompose lignin with the same efficiency.

\subsection{Notations}

Table 1. Notations.

\begin{tabular}{|c|c|c|}
\hline Symbol & Description & Unit \\
\hline $\mathrm{t}$ & $\begin{array}{c}\text { the number of days after fungi start to } \\
\text { decompose }\end{array}$ & day \\
\hline $\mathrm{T}$ & temperature in the environment & ${ }^{\circ} \mathrm{C}$ \\
\hline $\mathrm{W}$ & moisture in the environment & $\mathrm{MPa}$ \\
\hline $\mathrm{ER}$ & extension rate of the hyphae per day & $\mathrm{mm} / \mathrm{d}$ \\
\hline $\mathrm{DR}$ & $\begin{array}{c}\text { decomposition rate or the ratio of the } \\
\text { decomposed lignin to the total lignin } \\
\text { in t days }\end{array}$ & $\%$ \\
\hline
\end{tabular}

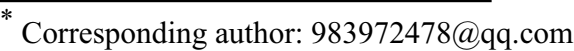




\begin{tabular}{|c|c|c|}
\hline $\mathrm{M}$ & $\begin{array}{c}\text { the ratio of the undecomposed lignin } \\
\text { to the total lignin in t days }\end{array}$ & $\%$ \\
\hline $\mathrm{MT}$ & $\begin{array}{c}\text { moisture tolerance. The difference } \\
\text { between a fungus' competitive } \\
\text { ranking and its moisture niche width. }\end{array}$ & $/$ \\
\hline$\beta$ & moisture weakening coefficient & $/$ \\
\hline $\mathrm{b}$ & decomposition ability coefficient & $/$ \\
\hline$\alpha$ & competitive coefficient & $/$ \\
\hline $\mathrm{r}$ & single colony radius & $\mathrm{mm}$ \\
\hline
\end{tabular}

\section{Model Establishment}

We built Colony Distribution-Decomposition Model, which was divided into 3 sub-models: single colony growth and extension model, decomposition model and competition model.

\subsection{Single Colony Growth and Extension Model}

We simplified the proliferation of fungi to two aspects: the extension and the thickening of hyphae. The thickening means the increase of cell number in per unit length of hypha.

Therefore, we assumed that a fungal colony is a standard circle ${ }^{[3]}$, and its colony expansion is only related with the extension of the hyphae. We use the extension rate (ER) to represent the growth rate of the fungi.

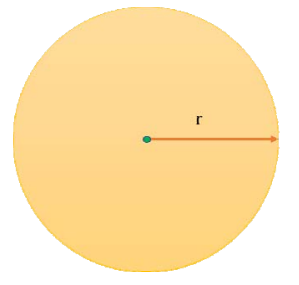

Fig. 1. Colony Abstract Illustration.

As shown in Fig. 1, when the fungus was initially inoculated, it started to expand around the inoculation point. So, the colony radius expansion rate was equal to the extension rate (ER).

$$
\frac{d r}{d t}=E R
$$

We found that ER was related with the temperature and humidity by learning the growth characteristics of fungi.

\subsubsection{The Influence of Temperature on ER}

We selected the ER of 34 fungi, and compare their ER at $10,16,22^{\circ} \mathrm{C}$.As shown in the Fig. 2, we found that ER changed with temperature and the change trends differed in different species. Most fungal ER increased as temperature increased, a small number of species increased at the beginning and then decreased as temperature increased. By contrast, very few species decreased at the beginning and increased afterwards.

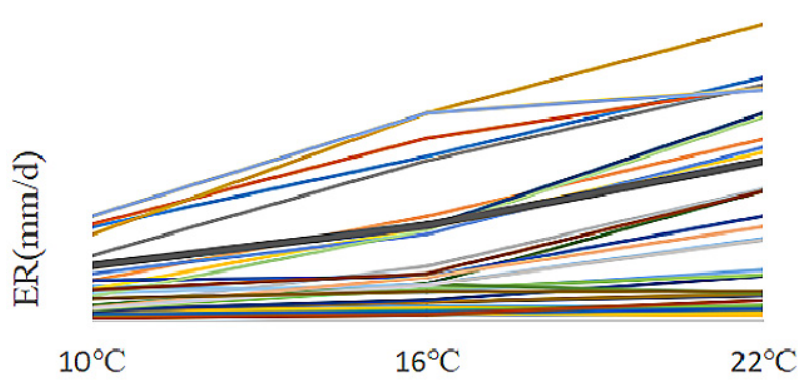

Fungus species

Fig. 2. Extension rate-temperature in different species.

According to the reference [4], we obtained the influence curve of temperature on fungal growth rate, which was shown in Fig. 3. As the temperature increased, extension rate changed. There are three critical temperature $T_{1}, T_{2}, T_{3}$, dividing the entire curve into 4 regions I -IV (Fig. 3).

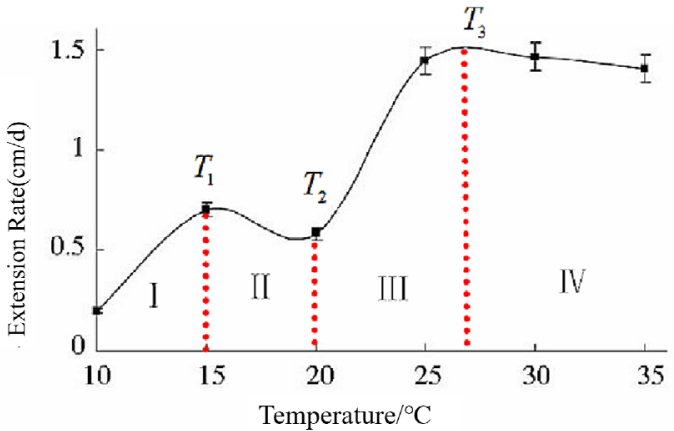

Fig. 3. Influence curve of temperature on fungal growth rate.

ER was linearly changed with temperature in individual region. We searched for the optimum temperature $\left(\mathrm{T}_{\mathrm{m}}\right)$ for 34 fungal and the extension rate $\left(E R_{m}\right)$ under the temperature. Data $E_{10}, E_{16}, E R_{22}$ and $\mathrm{ER}_{\mathrm{m}}$ were used to get the following formula.

$$
E R(T)= \begin{cases}\frac{16-T}{16-10} \mathrm{ER}_{10}+\frac{T-10}{16-10} \mathrm{ER}_{16} & (T \leq 16) \\ \frac{22-T}{22-16} E R_{16}+\frac{T-16}{22-16} E R_{22} & \left(1 C_{\zeta}<T \leq 22\right) \\ \frac{T_{m}-T}{T_{m}-22} E R_{22}+\frac{T-22}{T_{m}-22} E R_{m} & \left(22<T \leq T_{m}\right) \\ \frac{T-T_{3}}{T_{m}-22} E R_{22}+\frac{2 T_{3}-T-22}{T_{m}-22} E R_{m} & \left(T_{m}<T\right)\end{cases}
$$

Considering some optimum temperature being lower than $22^{\circ} \mathrm{C}$, the model was further modified as follows.

$$
E R(T)= \begin{cases}\frac{16-T}{16-10} \mathrm{ER}_{10}+\frac{T-10}{16-10} \mathrm{ER}_{16} & (T \leq 16) \\ \frac{T_{m}-T}{T_{m}-22} E R_{16}+\frac{T-16}{T_{m}-22} E R_{m} & \left(16<T \leq T_{m}\right) \\ \frac{22-\mathrm{T}}{22-T_{m}} E R_{m}+\frac{T-22}{22-T_{m}} E R_{22} & \left(T_{m}<T\right)\end{cases}
$$

\subsubsection{The Influence of Moisture on ER}

The optimum humidity varies among different fungi. As fungus is at its optimum moisture, the highest growth rate was achieved. At the maximum or minimum of moisture, only half of the fungal community can maintain its fastest growth rate. Thus, moisture impacted 
the percentage of individuals with the largest ER, which means moisture weaken ER at a certain temperature.

Moisture weakening coefficient $\beta$ represents the reduction degree of the humidity for the ER at a certain temperature.

The definition of Moisture Niche Width is: The difference between the maximum and minimum moisture levels in which half of a fungal community can maintain its fastest growth rate. Since the ratio of fungal community containing maximum rate at the minimum or maximum moisture level is $50 \%$, the average ER is $75 \%$ of the maximum rate and $\beta$ is equal to 1 at the optimum moisture.

We considered the geometric average of maximum moisture and minimum moisture as optimum moisture $w_{\mu}$, which is,

$$
w_{\mu}=\sqrt{w_{l} w_{h}}
$$

Assuming $\beta$ varied linearly with moisture, we established the following formula.

$$
\beta(w)= \begin{cases}\frac{\beta_{l}}{w_{l}} w & \left(0<w \leq w_{l}\right) \\ \frac{\beta_{\mu}-\beta_{l}}{w_{\mu}-w_{l}}\left(w-w_{\mu}\right)+\beta_{l} & \left(w_{l}<w \leq w_{\mu}\right) \\ \frac{\beta_{\mu}-\beta_{h}}{w_{\mu}-w_{h}}\left(w-w_{\mu}\right)+\beta_{h} & \left(w_{\mu} \leq w\right)\end{cases}
$$

Where $w_{h}$ represents maximum moisture and $w_{l}$ represents minimum moisture.

Considering the comprehensive influence of temperature and humidity, we established Equation (6) to describe the relationship of ER with temperature and moisture.

$$
E R=\beta(w) E R(T)
$$

\subsection{Decomposition Model}

\subsubsection{Decomposition-Logistic Curve}

We assumed that fungus grew on a ground litter with uniform lignin, and the initial content of lignin per unit area is $100 \%$.

The decomposition efficiency of each fungus is same, thus the slope of the lignin remaining rate curve is in proportion to the number of cells per unit volume. Considering the limited resources, the number of fungi would experience rapid increasing stage due to rapid proliferation, slower proliferation stage due to intraspecies competition, and eventually extinction stage due to exhaustion of supply. So Logistic equation was used to model the decomposition process of lignin (Fig. 4).

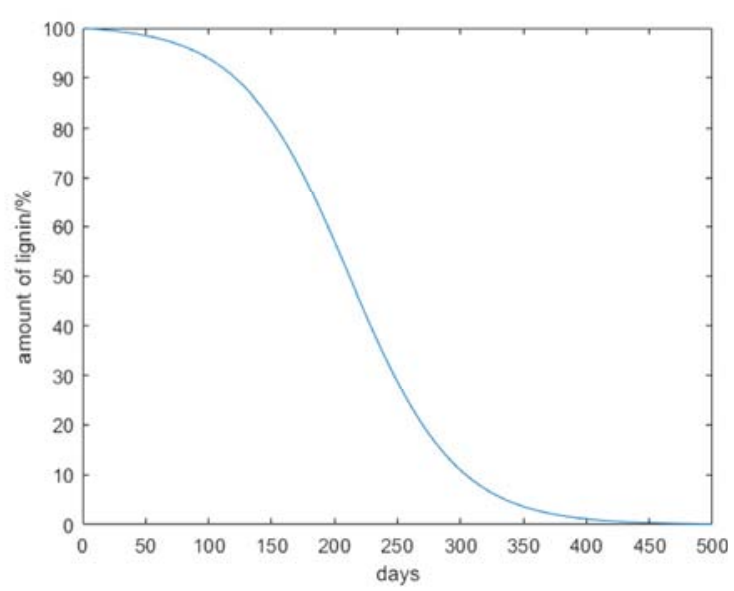

Fig. 4. Decomposition-Logistic Curve

The mathematical equation is,

$$
M(t)=\frac{K}{1+e^{a+b t}}
$$

Where, $\mathrm{M}(\mathrm{t})$ represents the undecomposed rate of lignin, $\mathrm{K}$ is a constant. To make sure $\mathrm{M}(0)=100 \%$, we define $K=1+e^{a}$; a is a constant. b is the characteristic value of the curve, which is related to the type of fungus and reflects the decomposition ability of fungus.

Based on the formula 7, we obtained the daily decomposition rate of lignin as follows :

$$
M^{\prime}(t)=\frac{\left(1+e^{a}\right) b e^{a+b t}}{1+e^{a+b t}}
$$

When $\mathrm{t}=0$, the number of cells is small, so the decomposition rate is close to 0

$$
M^{\prime}(0)=\frac{\left(1+e^{a}\right) b e^{a}}{1+e^{a}}=b e^{a} \rightarrow 0
$$

In the engineering application of Logistic Model, the constant a ranged from -3 to -5 . We defined $a=-5$ according to experience.

So, the final Decomposition-Logistic Curve is

$$
M(t)=\frac{1+e^{-5}}{1+e^{-5+b t}}
$$

\subsubsection{The Influence of Moisture and Temperature on $D R$}

According to reference ${ }^{[1]}$, decomposition rate (DR) is related with fungal characteristics.

Table $2^{[1]}$. The relationship between decomposition rate and fungal characteristics.

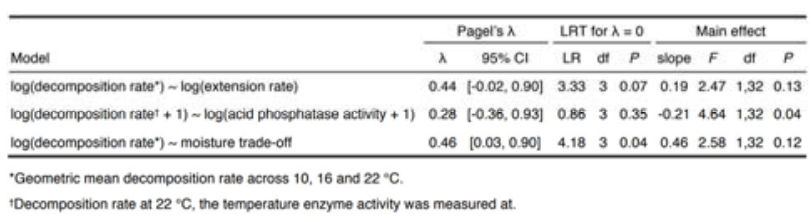

Moisture (MT) and extension rate (ER) are important to decomposition rate (DR). According to the result of phylogenetic least squares regression above, we obtained the Equation (11) 


$$
\log D R=a_{0}+a_{1} \log E R+a_{2} M T
$$

where ai $(i=0,1,2)$ is parameter, which is a constant.

According to Single Colony Growth and Extension Model, we can get the relationship $E R=\beta(w) E R(T)$. So $\mathrm{DR}$ is also relate to moisture and temperature.

Moisture tolerance is the difference between a fungus' competitive ranking and its moisture niche width, which is only related to fungal species. Therefore, for a specific kind of fungus it is a constant which means Moisture tolerance (difference of each isolate's competitive ranking and their moisture niche width, both scaled to $[0,1])$. Thus, for fungus(i), we can describe it as

$$
\log D R=\log E R+C_{i}
$$

Where $\mathrm{C} i$ is a constant.

Obviously, DR and ER are linearly correlated. Since ER was linearly changed with temperature and moisture, DR was also changed linearly with $\mathrm{T}$ and $\mathrm{w}$.

$$
D R=\beta(w) D R(T)
$$

Where,

$$
\begin{aligned}
& D R(T)= \begin{cases}\frac{D R_{16}-\mathrm{D} R_{10}}{16-10}(T-10)+\mathrm{D} R_{10} & (10 \leq T \leq 16) \\
\frac{D R_{22}-\mathrm{D} R_{16}}{22-16}(T-16)+\mathrm{D} R_{16} & (16<T \leq 25)\end{cases} \\
& \beta(w)= \begin{cases}\frac{\beta_{l}}{w_{l}} w & \left(0<w \leq w_{l}\right) \\
\frac{\beta_{\mu}-\beta_{l}}{w_{\mu}-w_{l}}\left(w-w_{\mu}\right)+\beta_{l} & \left(w_{l}<w \leq w_{\mu}\right) \\
\frac{\beta_{\mu}-\beta_{h}}{w_{\mu}-w_{h}}\left(w-w_{\mu}\right)+\beta_{h} & \left(w_{\mu} \leq w\right)\end{cases}
\end{aligned}
$$

$\mathrm{DR}_{10} 、 \mathrm{DR}_{16} 、 \mathrm{DR}_{22}$ represent the decomposition rate of a certain species at $10^{\circ} \mathrm{C}, 16^{\circ} \mathrm{C}, 22^{\circ} \mathrm{C}$.

\subsection{Competition Model}

Since the source of lignin is limited, there must be competition within a system. The competition can be divided into intra-specific competition and inter-specific competition.

Intra-species competition can be understood as the number of fungi shows the effect of first increasing and then decreasing and eventually leading to zero with the gradual decline of nutrients. Therefore, intra-specific competition has been reflected by the decompositionlogistic curve of the previous model.

An inter-species competition model was established as follows. When two colonies of different species coexist, competition occurs in the overlapping area. As shown in Fig. 5, we enlarged the unit area in the overlapping area. Each spot represents a cell of hypha. We assumed that in unit area, the hyphae of various species were mixed and staggered, and they grew and competed together in this unit area.

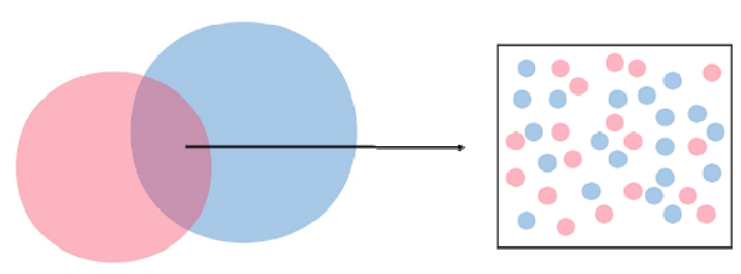

Fig. 5. Competition Abstract Illustration

It was assumed that the coexistence of two colonies exerted neglectable impact on the extension rate of the colony radius. The competitiveness is mainly reflected by the ability of decomposing lignin per unit area, which is $b$ in the decomposition -logistic curve.

When there is only one species with no inter-species competition. The decomposition ability of fungus per unit area is reflected in $b$.

When there are two kinds of fungus $i$ and $j$ in this unit area. If there is no inter-species competition, the lignin decomposition ability would be the sum of two fungal decomposition ability.

If we consider inter-species competition, they will inhibit each other' $\mathrm{s}$ decomposition rate. The more competitive species would eventually eliminate the less competitive species.

Thus, competitive coefficient $\alpha$ was used to reflect the inhibitory effect of a highly competitive species on a less competitive species.

$$
\alpha=\frac{b_{i}}{b_{j}}, \quad\left(b_{i}>b_{j}\right)
$$

Along with time, less competitive species $\mathrm{j}$ will be eliminated due to the insufficient nutrition, and its contribution to the total decomposition ability $b$ will become less and less until it disappears completely. Therefore, after $\mathrm{t}$ days, the competition starts, the total decomposition ability of the fungi per unit area can be expressed as,

$$
b=b_{i}+\alpha^{-t} b_{j}
$$

When $\mathrm{n}$ types of species compete together $(n \geq 2)$,

$$
\begin{gathered}
b_{\max }=\max \left\{b_{i}\right\}(i=1,2 \cdots n) \\
\alpha_{i}=\frac{b_{\max }}{b_{i}}(i=1,2 \cdots n) \\
b=\sum_{1}^{n} \alpha_{i}^{-t_{i}} b_{i}(i=1,2 \cdots n)
\end{gathered}
$$

Where $\mathrm{ct}_{\mathrm{i}}$ means the days species $\mathrm{i}$ enters this unit area.

\subsection{Colony Distribution-Decomposition Model}

Emerge three sub-models to a comprehensive model: Colony Distribution-Decomposition Model.

The extension rate of the hyphae is affected by the external environmental factors $\mathrm{T}$ and $\mathrm{w}$. The moisture tolerance (MT) of the fungus is affected by the internal factors including competitive ability and moisture niche width. These two factors together affect the decomposition rate (DR), and at the same time DR is 
also affected by the competitive coefficient. The remaining lignin in a litter can be reflected by DR. Finally, the external conditions, colony growth and hyphal extension and decomposed lignin in the litter are linked together.

In order to represent it in MATLAB, we construct a square to simulate the ground litter, which contains 500 $\times 500$ lattice. A lattice represents a unit area. We arrange randomly any species of fungi into lattice and the colonies begin to expand with this initial point as the origin. When external environment is changing, we can calculate ER through the constructed formula to simulate fungal reaction to the changing climate.

Each lattice initially has 100\% lignin, and the lignin remaining in each lattice covered by the colony decreases with time according to the decomposition model. If multiple fungi appear in the same lattice at the same time, the competition model was used to change the competitive ability coefficient $b$ and apply the new one to the decomposition-logistic curve to change the decomposition rate.

Thus, the Colony Distribution-Decomposition Model has been constructed completely.

\section{Results \& Discussion}

Based on Colony Distribution-Decomposition Model, we investigated the relevant factors to the decomposition rate of fungi.

\subsection{The Influence of the Interaction between Different Species}

We inoculated multiple fungi whose extension rate and moisture resistance were different into the same simulated square and used our model to describe the ground litter and the decomposition rate of woody fibers to represent the interaction between multiple fungi.

According to the experimental results (Fig 6,7), under the action of a single fungus, only about $10 \%$ of the total lignin has been decomposed in 122 days. Under the mixed action of multiple fungi, the highest decomposition rate reaches nearly $50 \%$. The contribution of lattices which have the same remaining lignin is no longer a standard circle, and has a certain randomness, which is related with the positional relationship of the inoculated 34 kinds of fungi.

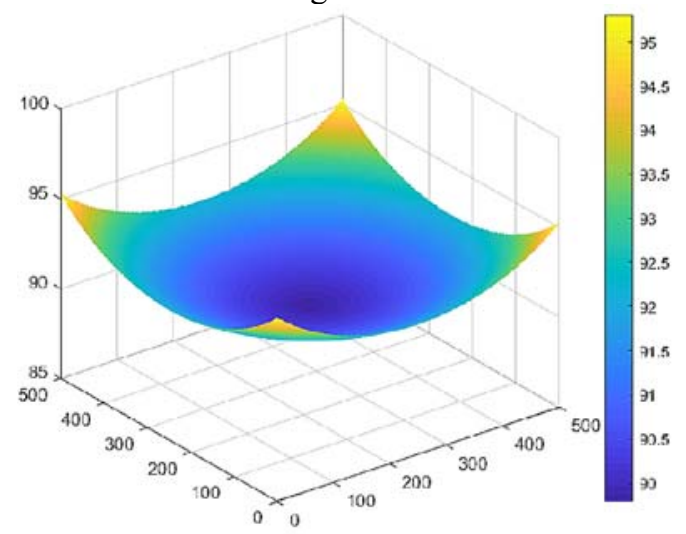

Fig. 6. single fungus decomposition effect

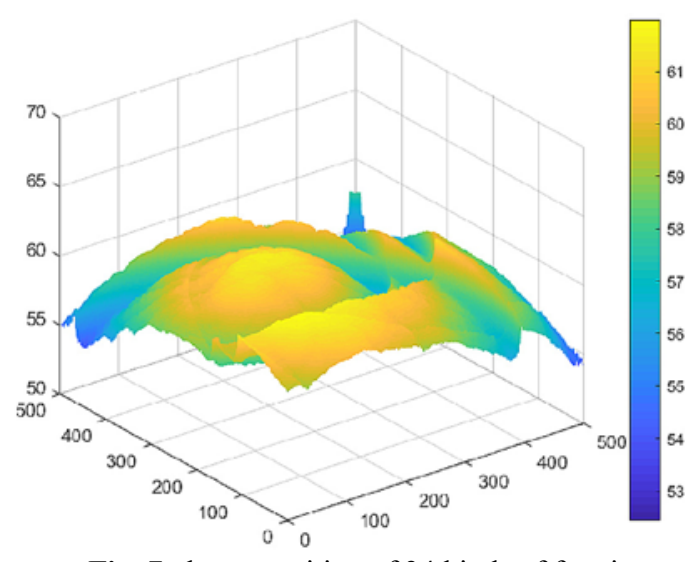

Fig. 7. decomposition of 34 kinds of fungi

\subsection{The Impact of Environmental Fluctuations}

We examined the sensitivity of fungal decomposition effect to rapid fluctuations in the environment. From the long-term perspective, regardless of day - night or seasonal fluctuations, the environment would reach a steady state. Temperature and moisture were fixed values, but different stable values exerted different impact on the decomposition efficiency of fungi. In the short term, due to the alternation of day and night or the changes of four seasons, the temperature and moisture would fluctuate rapidly and affect the decomposition rate. The results are shown in Fig. 8 and 9.

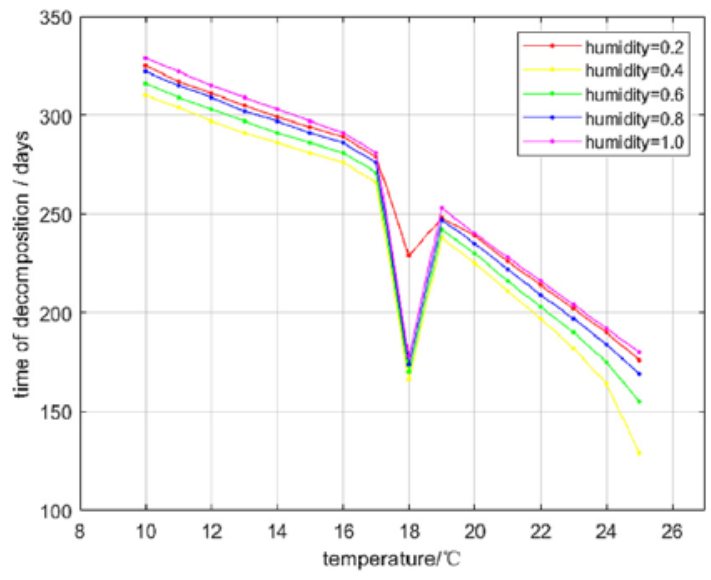

Fig. 8. long-term effect

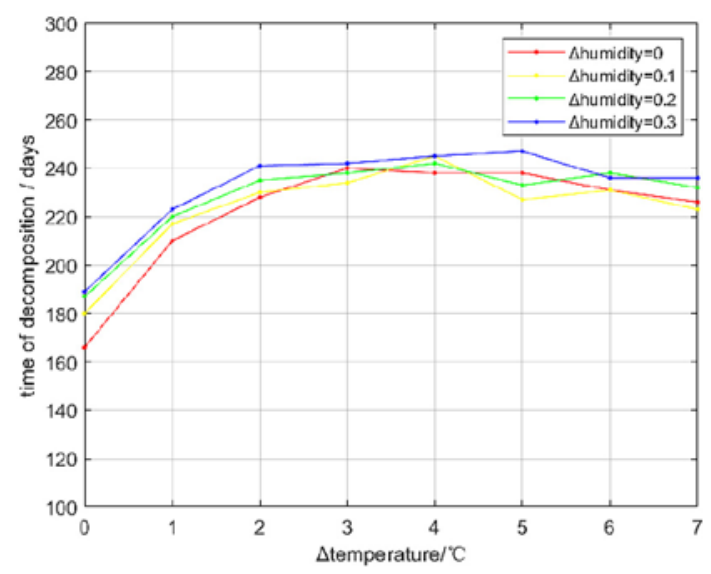

Fig. 9. short-term effect 
Considering the long-term environmental impact, $18^{\circ} \mathrm{C}$ and $0.4 \mathrm{MPa}$ are most suitable for the growth of this group of fungi. If the long-term trend of the local atmosphere deviates from this value, the growth of local fungi and the decomposition effect wouldl be inhibited. If short-term environmental impacts are considered, the introduction of certain environmental fluctuations (such as rapid changes in temperature and humidity) would have an adverse effect on the growth of the local fungi and the decomposition effect. The more severe the fluctuation, the adverse effect would be more significant.

\subsection{Fungus Environmental Adaptability Study}

We analyzed the environmental adaptability of different species under different climatic conditions. And predict the dominant and inferior species under four climatic conditions: arid, semi-arid, temperate, and tropical rainforest.

The four environmental conditions were simulated by our model, and we recorded the maximum of each fungus to indicate the adaptation to such kind of environment. The larger the relative amount is, the stronger the adaptability is in this climate type.

The adapted or unadapted species in the four different climates are as follows, and only the first three are shown (Table 3).

Table 3. The adapted and unadapted species in 4 climate types.

\begin{tabular}{|c|c|c|}
\hline climate & adapted species & uadapted species \\
\hline \multirow{3}{*}{ Arid } & $\begin{array}{c}\text { Armillaria_tabescens_T } \\
\text { JV93_261_A1E }\end{array}$ & $\begin{array}{c}\text { Laetiporus_conifericola } \\
\text { HHB15411_C8B }\end{array}$ \\
\hline & $\begin{array}{c}\text { Hyphoderma_setigerum } \\
\text { HHB } 12156 \mathrm{~B} 3 \mathrm{H}\end{array}$ & Armillaria_gallica_EL8 \\
\hline & $\begin{array}{c}\text { Lentinus_crinitus_PR20 } \\
58 \text { C1B }\end{array}$ & $\begin{array}{c}\text { Armillaria_gallica_FP10 } \\
2534 \text { A5 A }\end{array}$ \\
\hline \multirow{3}{*}{ semi-arid } & Armillaria_sinapina_PR & $\begin{array}{c}\text { Laetiporus_conifericola } \\
\text { HHB15411 C8B }\end{array}$ \\
\hline & \begin{tabular}{|c|} 
Fomes_fomentarius_TJ \\
V93_7_A3E \\
\end{tabular} & $\begin{array}{c}\text { Armillaria_gallica_FP10 } \\
\text { 2531_C6D }\end{array}$ \\
\hline & $\begin{array}{c}\text { Hyphoderma_setigerum } \\
\text { FP150263_B2C }\end{array}$ & $\underset{\text { A6E }}{\text { Armillaria_gallica_OC1 }}$ \\
\hline \multirow{3}{*}{ temperate } & $\begin{array}{c}\text { Armillaria_gallica_FP1 } \\
\text { 02531_C6D }\end{array}$ & Armillaria_sinapina_PR9 \\
\hline & \begin{tabular}{|c|} 
Hyphodontia_crustosa_ \\
HHB13392_B7B
\end{tabular} & $\begin{array}{c}\text { Armillaria_tabescens_TJ } \\
\text { V93_261_A1E }\end{array}$ \\
\hline & $\begin{array}{c}\text { Laetiporus_conifericola } \\
\text { HHB15411_C8B }\end{array}$ & $\begin{array}{c}\text { Fomes_fomentarius_TJV } \\
937 \text { A3E }\end{array}$ \\
\hline \multirow{3}{*}{$\begin{array}{l}\text { tropical } \\
\text { rainforest }\end{array}$} & $\underset{\text { A66 }}{\text { Armillaria_gallica_EL8 }}$ & $\begin{array}{c}\text { Laetiporus_conifericola } \\
\text { HHB15411_C8B }\end{array}$ \\
\hline & $\begin{array}{c}\text { Armillaria_gallica_FP1 } \\
02534 \text { A5 A } \\
\end{array}$ & $\begin{array}{c}\text { Mycoacia_meridionalis_ } \\
\text { FP150352_C4E }\end{array}$ \\
\hline & $\begin{array}{c}\text { Armillaria_gallica_FP1 } \\
02542 \text { A5B }\end{array}$ & $\begin{array}{c}\text { Phlebia_acerina_MR428 } \\
\text { 0_B9G }\end{array}$ \\
\hline
\end{tabular}

\subsection{Biodiversity Analysis}

We inoculated 34 fungi separately and measured the time to achieve the lignin decomposition rate of $99 \%$ under 9 types of environmental fluctuations and inoculated multiple fungi.
Fig 10 shows that under the same environmental disturbance, the decomposition of multiple fungal communities is more efficient and stable. Therefore, biodiversity is not only conducive to increase the efficiency of decomposition, but also is conducive to the colony resisting environmental fluctuations and enhancing the stability of the system.

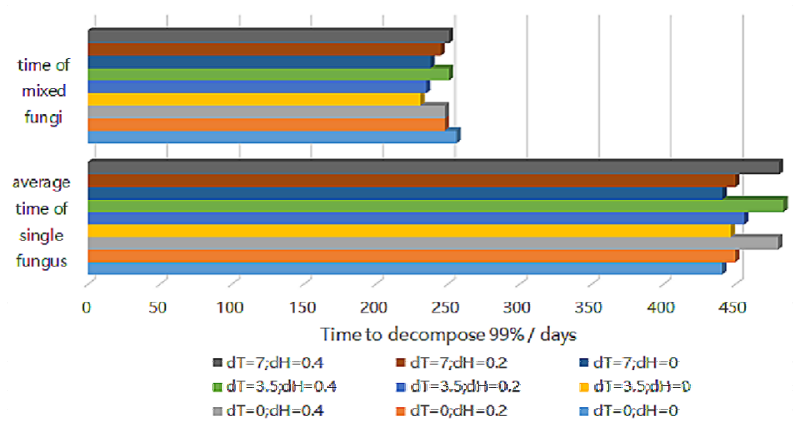

Fig. 10. Environmental fluctuations on decomposition time

In order to accurately measure the fluctuations in decomposition efficiency caused by environmental disturbances, we calculated the standard deviations of the decomposition time of 34 independent and multiple fungi, which is

$$
\sigma_{\text {isolate }}=16.55, \quad \sigma_{\text {mixed }}=7.86
$$

Normalize the decomposition time separately.

$$
{\sigma_{\text {isolate }}}^{*}=0.034, \sigma_{\text {mixed }}{ }^{*}=0.029
$$

Two groups of results indicated that under the same environmental disturbance, the decomposition of multiple fungi communities was more efficient and stable.

\section{Conclusions}

We built a Colony Distribution-Decomposition Model to describe the decomposition rate of ground litter and woody fibers through the interaction of different fungi.

For the interaction of different species, our result showed that multiple strains of fungi changed the distribution of the remaining amount of decomposed matter and increased the decomposition rate.

For the sensitivity of fungi to environmental changes in the short-term and long-term periods, we found that rapid fluctuations in the environment reduced decomposition efficiency.

For the adapatabilities of various species to different climate types, we achieved the results of the dominant and disadvantaged species in arid, semi-arid, temperate, and tropical rain forests climate.

For the role of biodiversity in the breakdown of the litter, we drew a conclusion that biodiversity could improve the decomposition efficiency and resistance to environmental changes.

In a word, our model has the advantages of comprehensiveness, stability, rationality, and wide application, which is a feasible and reasonable model to 
investigate the interaction between multiple fungi and describe the relationship between fungal activity and decomposition.

\section{References}

1. Nicky Lustenhouwer,Daniel S. Maynard,Mark A. Bradford,Daniel L. Lindner,Brad Oberle,Amy E. Zanne,Thomas W. Crowther. A trait-based understanding of wood decomposition by fungi[J]. Proceedings of the National Academy of Sciences,2020(prepublish).

2. Daniel S. Maynard,Mark A. Bradford,Kristofer R. Covey,Daniel Lindner,Jessie Glaeser,Douglas A. Talbert,Paul Joshua Tinker,Donald M. Walker,Thomas W. Crowther. Consistent trade-offs in fungal trait expression across broad spatial scales[J]. Nature Microbiology,2019,4(5).

3. LU Yu-xin YANG Xue-ying MI Yang LIU Kai-hui DENG Bai-wan ZHAO Hang-ke Biological Characteristic Research of Halophilic Fungus Aspergillus montevidensis [J].Southwest China Journal of Agricultural Sciences,2020,33(07):14411448.

4. YAN Ju-fen QI Ning-bo WANG Su-ping GAI Li-li YANG Shu-lin, YAN Ju-fen QI Ning-bo WANG Su-ping GAI Li-li YANG Shu-lin[J].China Biotechnology, ,2013,33(10):51-58. 